\title{
Correlative Microbially-Assisted Imaging of Cellulose Deconstruction with Electron Microscopy
}

\author{
Alejandra Londono-Calderon ${ }^{1}$, Wan Sun ${ }^{2}$, Zengyi Shao ${ }^{1,2}$, Daan Hein Alsem ${ }^{3}$, and Tanya Prozorov ${ }^{1}$ \\ ${ }^{1}$ US DOE Ames Laboratory, Division of Materials Science and Engineering, Ames, IA 50011, USA. \\ ${ }^{2}$ Iowa State University, Department of Chemical and Biological Engineering, Ames, IA 50011, USA. \\ ${ }^{3}$ Hummingbird Scientific, Lacey, WA, USA
}

Microscopy aided in many important discoveries in life sciences by providing structural information about cellular compartments. Among the many types of analytical microscopy, transmission electron microscopy (TEM) is a widely used technique to characterize matter at high spatial resolution. However, electron microscopy analysis of biological samples is traditionally restricted to working with dried and prepared specimens devoid of their natural environment, to comply with the strict vacuum requirements of the instruments. With the recent developments in the field of in situ electron microscopy with fluid cell, it is finally possible to image proteins, macromolecular complexes, viable bacterial cells and cellular compartments in their natural, fully hydrated state [1]. This provides an opportunity to probe the intact microorganisms, map the specific chemical composition, and monitor multiple metabolic processes within and between intact cells in their native environment. Efficient biomass degradation in nature requires synergistic efforts of an ensemble of microbes that secrete or surface-display cellulolytic and lignolytic enzymes with appropriate ratios and spatial arrangement. Using targeted microbes and enzymes produced by an artificial microbial community mimicking this natural phenomenon, we are able to obtain first insight on cellulose deconstruction in a model system.

In this work, we used correlative high angle annular dark field scanning transmission electron microscopy - scanning electron microscopy - fluorescent microscopy HAADF-STEM-SEM-FM imaging to follow microbially-assisted deconstruction of cellulose. We use a continuous flow fluid cell platform that is compatible with both FEI Tecnai (S)TEM and FEI Teneo SEM instruments. After performing a number of calibration tests, the fluid cell platform is being used for the visualization of complex biomass features and microbial cells in a fully hydrated state, while providing structural information across a wide range of scales. Initial experiments were carried out using cells of modified, surface cellulase-expressing Saccharomyces cerevisiae grown to different optical densities, and left incubating with carboxymethyl-cellulose (CMC) at $30{ }^{\circ} \mathrm{C}$ overnight under gentle shaking [2-3].

Figure 1 shows DNS biochemical assay and the glucose standard for $S$. cerevisiae with and without CMC. The displayed enzyme degraded CMC to sugar monomers; an increase in biomass hydrolysis is observed as a consequence of higher optical cell density. Figure 2(a-b) show post-mortem HAADFSTEM images of 5OD cells incubated with and without $\mathrm{CMC}$ on $\mathrm{aC}-\mathrm{Cu}$ grid. The red arrows on (b) marked hydrolyzed CMC on the yeast surface. Fully hydrated cells and fragments of partially degraded biomass were analyzed by the use of the fluid cell platform as observed on (c). Our approach allows imaging of cellulose deconstruction under mild conditions. We envision that our results will bring us closer to understanding of this technologically important process.

Current experiments involve immobilization of CMC on the surface of SiN windows and incubating the immobilized cellulosed overnight with a small number of $S$. cerevisiae cells, followed by the in situ imaging in liquid phase. We pay attention to controlling the electron dose to the specimen in both 
electron microscopes and careful assessment of microbial cell viability exposed to electron beam under the two sets of operating voltages. Use of the fluid cell platform enables correlative multimodal quantitative imaging approach and allows utilization of latest advances in chemical composition mapping and high-resolution microscopy of cellulose deconstruction by the microbial cells in their native environment [4].

References:

[1] D. B. Peckys, et al, Biophysical Journal 100 (2011), p. 2522

[2] B. C. King, et al, Biotechnology and Bioengineering 102 (2009), p. 1033.

[3] J. Lian, et al, Nature Communications 8 (2017), p. 1688.

[4] This work was supported by the U.S. Department of Energy (DOE), Office of Science, Basic Energy Research, Office of Biological and Environmental Research. The research was performed at the Ames Laboratory, which is operated for the U.S. Department of Energy by Iowa State University under Contract No. DE-AC02-07CH11358.

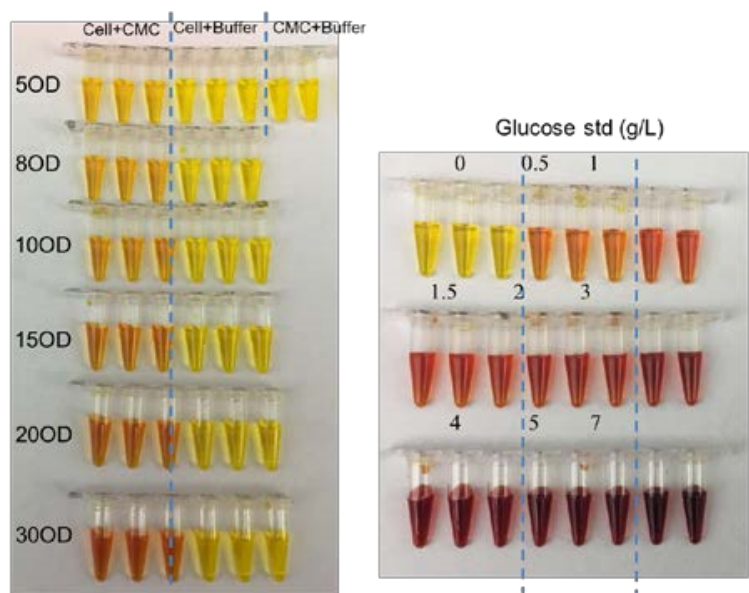

Figure 1. (Left) Reducing sugar quantification (DNS assay) of modified Saccharomyces cerevisiae cells with CMC in sodium acetate. (Right) Glucose standard.

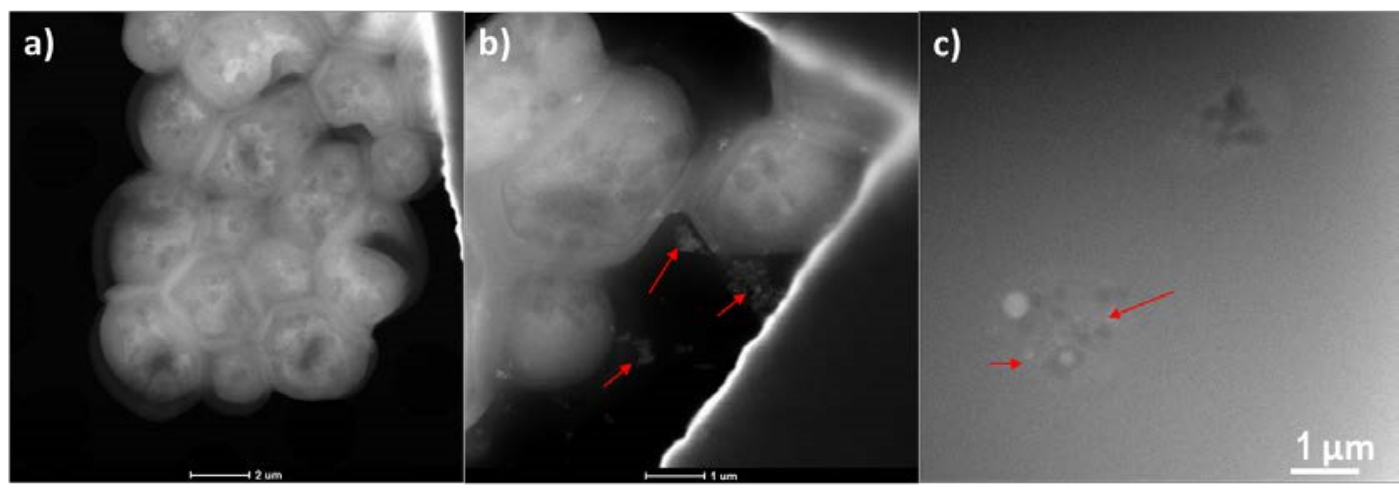

Figure 2. Small colony of yeast (a) before and (b) after incubation with CMC. Cells on part (b) exhibits a "decoration" in close proximity to their surface (marked with red arrows), the marked features are likely hydrolyzed fragments of biomass; c) S. cerevisiae cells in liquid phase appear intact. Marked with red arrows are small fragments corresponding to the "decoration" in b). 